\title{
Alfa-fetoproteína sérica en la predicción de parto pretérmino
}

\author{
José Urdaneta Machado, $P h D^{1}$, Selene Leal ${ }^{2}$, José García, $P h D^{3}$, Maritza Cepeda de \\ Villalobos, $P h D^{3}$, Mery Guerra, $P h D^{3}$, Nasser Baabel Zambrano, $P h D^{3}$, Jaime Leal, 3 \\ Alfi Contreras Benítez.2 \\ ${ }^{1}$ Universidad del Zulia, Facultad de Medicina, Departamento de Morfofisiopatología. ${ }^{2}$ Cursante de Postgrado, División \\ de estudios para graduados, Facultad de Medicina, Universidad del Zulia. ${ }^{3}$ Universidad del Zulia, Facultad de Medicina, \\ Departamento de Obstetricia y Ginecología. Venezuela.
}

\section{RESUMEN}

Objetivo: Relacionar las concentraciones de la alfafetoproteína (AFP) sérica con la ocurrencia de parto pretérmino (PP) en embarazadas con y sin factores de riesgo (FR) atendidas en la Maternidad "Dr. Armando Castillo Plaza" en Maracaibo, Estado Zulia, Venezuela. Métodos: Investigación predictiva y comparativa aplicada, que incluyo 60 embarazadas con y sin FR para PP $(n=30$ para cada grupo), a las que se les determinó la AFP sérica entre las 24-28 semanas de gestación, siguiéndose hasta la culminación del embarazo. Resultados: Las concentraciones de AFP en gestantes con FR fueron significativamente mayores (341,35 $\pm 183,51$ vs. $238,63 \pm 110,46 ; p<0,05)$; asimismo, el diagnóstico de PP se efectuó en $43,33 \%$ de las gestantes con $\mathrm{FR}$ y en $13,33 \%$ sin $\mathrm{FR}(\mathrm{p}<0,05)$. Esta prueba presentó en gestantes con $\mathrm{FR}$ tasas más altas de sensibilidad $(77 \%)$, especificidad $(76 \%)$, valor predictivo positivo $(71 \%)$ o negativo $(81 \%)$, predicción total $(77 \%)$; razón de verosimilitud positiva $(3,27)$ y probabilidad post-test positiva $(71 \%)$; mientras que la razón de verosimilitud negativa $(0,30)$ y la probabilidad post-test negativa $(19 \%)$ fueron bajas, en tanto que el riesgo de PP fue significativo (OR [IC95\%]= 10,833 [1,961-59,835]; p<0,05). Conclusión: Existe una relación entre la elevación de las concentraciones de AFP y la presencia de PP, mostrando esta prueba tener una alta capacidad diagnóstica y poder predictivo para la ocurrencia de esta patología en embarazadas con FR.

\section{PALABRAS CLAVE: Alfa-fetoproteína, factores de riesgo, parto pretérmino}

\section{SUMMARY}

Objective: To relate the concentrations of alpha-fetoprotein (AFP) levels with the occurrence of preterm delivery $(\mathrm{PP})$ in pregnant women with and without risk factors (RF) attending Motherhood "Dr. Armando Castillo Plaza" in Maracaibo, Zulia State, Venezuela. Methods: This research is a predictive and comparative type, which included 60 pregnant women with and without RF for PP ( $n=30$ for each group), which were determined from serum AFP 24-28 weeks of gestation, being followed until completion of pregnancy. Results: The concentrations of AFP in pregnancies with RF were significantly higher (341.35 $\pm 183.51 \mathrm{vs.} 238.63 \pm$ $110.46, p<0.05$ ), also the diagnostic PP was performed in $43.33 \%$ of pregnant women with RF and non RF $13.33 \%(p<0.05)$. This test presented in RF pregnant with higher rates of sensitivity $(77 \%)$, specificity $(76 \%)$, positive predictive value $(71 \%)$ or negative $(81 \%)$, total prediction $(77 \%)$, positive likelihood ratio (3.27) and positive post-test probability $(71 \%)$, whereas the negative likelihood ratio $(0.30)$ and the post-test probability negative $(19 \%)$ were low, while the risk of PP was significant $(\mathrm{OR}[95 \% \mathrm{Cl}]=10.833$ [1.961 to 59.835]; 
$\mathrm{p}<0.05)$. Conclusion: There is a relationship between elevated AFP levels and the presences of PP, showing this test have high diagnostic accuracy and predictive power for the occurrence of this disease in pregnant with RF.

\section{KEY WORDS: Alpha-fetoprotein, risk's factors, preterm delivery}

\section{INTRODUCCIÓN}

Anualmente alrededor del mundo nacen $13 \mathrm{mi}-$ llones de niños prematuros, la mayoría en países en desarrollo, donde el parto pretérmino (PP) constituye el componente principal de la morbi-mortalidad perinatal (1), aportando más del $70 \%$ de la morbi-mortalidad neonatal mundial, con una incidencia entre 7 - 12\% en los Estados Unidos de Norteamérica (2), donde al igual que en Europa, a pesar del incremento del uso de tocolíticos, su incidencia permanece sin cambios alrededor del $8 \%$ (3).

En Latinoamérica y el Caribe cada año mueren aproximadamente 135.000 niños debido a prematuridad, siendo la situación aún más grave en infantes con prematuridad extrema, entre quienes una quinta parte no supervive el primer año y hasta $60 \%$ de los supervivientes tiene discapacidades neurológicas (4). En Venezuela, en los últimos años, se ha observado que la tasa de nacimientos pretérminos ha ido aumentando de forma sostenida, coincidiendo con un repunte del embarazo adolescente, constituyendo un problema de salud pública de gran impacto (5). Según las últimas cifras oficiales gubernamentales reportadas las tasas de mortalidad neonatal oscilan entre 13,8 a 10,8 por mil nacidos vivos (6) y ha sido reportada una incidencia de PP del $9 \%$, asociada con $34,31 \%$ de mortalidad neonatal (7).

Actualmente han surgido diversos marcadores para la predicción del PP, a fin de superar las debilidades del examen obstétrico (8) y permitir así un diagnóstico oportuno y el establecimiento de un criterio de decisión para el correcto tratamiento (9). Entre ellas se encuentran la medición ecografía de cuello uterino $(10,11)$, estriol salival $(12)$, determinación de citoquinas o fibronectina fetal en secreciones vaginales $(13,14)$, y recientemente marcadores séricos como la alfa fetoproteína (AFP). Esta es una glucoproteína sintetizada principalmente por el hígado fetal normal, la cual se encuentra en altas concentraciones en el suero fetal y bajo circunstancias normales cantidades muy pequeñas pero medibles pasan a la circulación materna desde el compartimiento del líquido amniótico (1). Su producción se inicia en la cuarta semana de gestación y alcanza concentraciones máximas en la décima segunda semana, contribuyendo también en su producción el saco vitelino y el tubo digestivo (14).

La cuantificación de la AFP sérica se ha convertido en un complementario de gran valor para la obstetricia, representando un importante marcador bioquímico en el seguimiento y evaluación de los embarazos, describiéndose en las gestaciones simples un riesgo incrementando tanto para PP como otras complicaciones obstétricas (1517). Por tanto, surgió el propósito de relacionar las concentraciones de la AFP sérica con la ocurrencia de PP en embarazadas con y sin factores de riesgo (FR) atendidas en la Maternidad "Dr. Armando Castillo Plaza" de Maracaibo, Venezuela.

\section{PACIENTES Y MÉTODOS}

Investigación predictiva y comparativa, donde se seleccionaron de manera intencionada y no aleatoria a 30 embarazadas con FR para desencadenar PP (Grupo A) y 30 gestantes sin FR (Grupo B), atendidas en la consulta prenatal de la Maternidad "Dr. Armando Castillo Plaza". Dentro del Grupo A se incluyeron aquellas gestantes que refirieron FR para PP o que estuviesen registrados en sus expedientes clínicos y que cumplieron, adicionalmente, con los siguientes criterios: (a) edad gestacional entre $\geq 24$ semanas y $\leq 28$ semanas, estimada por la fecha de la última menstruación y confirmada por los datos obtenidos en una exploración ecográfica antes de las 20 semanas de gestación, (b) gestación única, y (c) edad comprendida entre los 19 y 35 años. Se excluyeron aquellas gestantes sintomáticas, con antecedentes de embarazos amenazados que fuesen sometidas a cerclajes uterinos, o que presenten comorbilidades como diabetes gestacional, malformaciones congénitas o polihidramnios. El Grupo B estuvo conformado por embarazadas sin FR para esta patología y con las características similares al grupo estudio, en cuanto a la edad, grupo étnico, nivel socioeconómico y procedencia.

A cada paciente se le solicitó el consentimiento informado por escrito para su inclusión en la investigación. Importa destacar que este estudio no representaba riesgo tanto para las pacientes involucradas como para los productos de sus embarazos, ni se vulneraron las normas éticas de la declaración de Helsinki. El protocolo fue previamente aprobado por el consejo Técnico de Postgrado de la Facultad de Medicina de la Universidad del Zulia y contó con el visto bueno de las autoridades sanitarias del hospital. 
Seguidamente se les realizó una evaluación obstétrica completa, que incluía la entrevista clínica para la identificación de los FR para PP. Posteriormente, previo ayuno de 8 horas se tomaron muestras sanguíneas $(5 \mathrm{ml})$ mediante punción de las venas antebraquiales con jeringa de plástico y agujas de acero inoxidable No. 21, recolectándose en tubos estériles secos y sin anticoagulante (Vacutainer() y colocadas durante diez minutos en una centrífuga a 4.000 RPM. Con el suero obtenido se determinaron los niveles de AFP, empleando el método inmunoensayo enzimático para su determinación cuantitativa, la cual está basada la técnica de Elisa sándwich haciendo uso del sistema de alta afinidad biotina estreptavidina, estas determinaciones se procesaron en el laboratorio de la institución.

Los valores utilizados como punto de referencia fueron los propuestos en Venezuela por Salazar y cols (18), los cuales presentan rangos de $82 \mathrm{ng} / \mathrm{ml}$ en el percentil 10 y $230 \mathrm{ng} / \mathrm{ml}$ en el percentil 90 con media de $133 \mathrm{ng} / \mathrm{ml}$. Todas las pacientes participantes en el estudio, independientemente de presentar resultados alterados o no, se siguieron prospectivamente en la consulta prenatal hasta la culminación del embarazo, de modo de poder detectar los casos de PP presentados en las gestantes con y sin FR.

Los datos generados se procesaron mediante el Paquete Estadístico para Ciencias Sociales (SPSS), versión 17, expresándose los resultados obtenidos mediante medidas de tendencia central, frecuencias absolutas y relativas, medias y desviación estándar (DE). Para comparar las concentraciones de AFP entre ambos grupos se utilizó la Prueba t de Student y para comparar las frecuencias de sus niveles elevados se utilizó la prueba del Chi cuadrado. Ambas con una significancia estadística del 95\% $(p<0,05)$.

Para determinar el valor diagnóstico de la AFP y su poder predictivo para PP, se calcularon las siguientes medidas, basadas tanto en el número de verdaderos positivos (VP) y negativos (VN) como de falsos positivos (FP) y negativos (FN): (a) Sensibilidad: (VP/VP+FN), (b) Especificidad: (VN/
VN+FP), (c) Valor predictivo positivo (VPP): (VP/ $\mathrm{VP}+\mathrm{FP}$ ), (d) Valor predictivo negativo (VPN): (VN/ $\mathrm{VN}+\mathrm{FN})$, (e) Razón de probabilidad positiva (LR+): (Sensibilidad / 1 - especificidad), (f) Razón de probabilidad negativa (LR-): (1 - sensibilidad / especificidad), (g) Precisión total: (VN+VP) / (todos los casos), (h) Odds: (Probabilidad / 1 - probabilidad) y (i) Odds post-test: (Odds pre-test x LR).

Para la estimación del riesgo, se procedió a calcular los Odds Ratio (OR) para cada factor identificado, mediante el análisis de regresión logística y la creación de tablas de contingencia $2 \times 2$ para determinar cada OR con sus respectivos intervalos de confianza 95\% (IC95\%), utilizándose la Prueba Exacta de Fisher para establecer su significancia estadística.

\section{RESULTADOS}

La edad promedio de las embarazadas del Grupo A fue de 26 años (rango: 23 - 29 años) y en el del grupo B fue de 27 años (rango: 24 - 30 años); mientras que la paridad fue de 2 en ambos grupos. La edad gestacional al momento de la determinación de la AFP para ambos grupos fue en promedio de 26 semanas; mientras que la edad gestacional al nacimiento se situó alrededor de las 39 semanas. No hubo diferencias estadísticamente significativas entre ambos grupos (Tabla I).

En cuanto a los FR para PP (Tabla II), prevalecieron las infecciones vaginales $(60 \%)$ y urinarias $(46,67 \%)$, el consumo de café durante la gestación $(36,67 \%)$, adolescencia $(40 \%)$, anemia (30\%) y antecedentes de PP (26,67\%); otros factores de riesgo detectados en menor frecuencia fueron la desnutrición, redes sociales ilimitadas, edad materna avanzada, miomatosis uterina y consumo de drogas ilícitas (cannabis). Respecto a las concentraciones de AFP (Tabla III) se encontró que las pacientes del grupo "A" presentaron significativamente niveles más elevados de AFP $(341,35 \pm$ $183,51$ vs. $238,63 \pm 110,46 ; p<0,05)$.

Tabla I

CARACTERÍSTICAS DE LA MUESTRA

\begin{tabular}{lccr}
\hline Variables & $\begin{array}{c}\text { Grupo A } \\
\text { *Media } \pm \text { DE }\end{array}$ & $\begin{array}{c}\text { Grupo B } \\
\text { *Media } \pm \text { DE }\end{array}$ & Valor $\mathrm{p}^{\star *}$ \\
\hline Edad & $26,35 \pm 03,51$ & $27,63 \pm 03,46$ & 0,160 \\
Paridad & $01,89 \pm 01,12$ & $02,23 \pm 00,65$ & 0,155 \\
EG al momento de la prueba (semanas) & $26,34 \pm 01,63$ & $26,79 \pm 01,25$ & 0,235 \\
EG al nacimiento (semanas) & $38,62 \pm 02,14$ & $39,02 \pm 01,84$ & 0,440 \\
\hline
\end{tabular}

Grupo A: con factores de riesgo. Grupo B: sin factores de riesgo. EG: edad gestacional. n: 30 para cada grupo.

${ }^{\star}$ Comparación de medias por T de Student. ${ }^{\star \star} p<0,05$ valor significativo. 
Tabla II

FACTORES DE RIESGO DE PARTO PRETÉRMINO

\begin{tabular}{lcc}
\hline Análisis & $\mathrm{n}$ & $\%$ \\
\hline Hábito cafeíco & 11 & 36,67 \\
Drogas ilícitas & 1 & 3,37 \\
Redes sociales ilimitadas & 6 & 20,00 \\
Adolescencia & 12 & 40,00 \\
Edad materna avanzada & 5 & 16,67 \\
Desnutrición & 6 & 20,00 \\
Antecedente de parto pretérmino & 8 & 26,67 \\
Anemia & 9 & 30,00 \\
Miomatosis uterina & 2 & 6,67 \\
Infecciones urinarias recurrentes & 14 & 46,67 \\
Infecciones vaginales & 18 & 60,00
\end{tabular}

Grupo $A=30$

Al término de la gestación la frecuencia de parto pretérmino fue de 13 casos $(43,33 \%)$ en las gestantes que presentaban FR y 4 casos $(13,33 \%)$ entre las gestantes del grupo B; diferencia significativa $\left(x^{2}=5,253 ; p=0,021\right)$. Asimismo, las pacientes con FR para PP y niveles elevados de AFP presentaron un riesgo significativo de diez veces más probabilidad de presentar esta complicación (OR [IC95\%]= $10,833$ [1,961-59,835]; $p<0,05)$; mientras las gestantes del grupo B no mostraron tener mayor probabilidad de presentarlo (Tabla IV).

Respecto a la capacidad diagnóstica de la AFP en la predicción del PP, las concentraciones elevadas de este marcador en el grupo de gestantes con FR mostraron una mejor capacidad diagnóstica y poder predictivo que en el otro grupo, caracterizada por tasas altas de sensibilidad, especificidad, VPP, VPN y predicción total; sin embargo, en el grupo B las concentraciones elevadas de AFP presentaron una alta sensibilidad y VPN para la predicción del PP (Tabla V). Asimismo, en el grupo A la prueba mostró una mayor razón de $\mathrm{LR}+\mathrm{y}$ una menor razón de LR-, lo cual es indicativo de una mejor capacidad para predecir la presencia de enfermedad (Tabla $\vee$ y Figura 1); a partir de estas razones se pudo calcular la probabilidad post-prueba, mostrando esta prueba en el grupo A una alta probabilidad de PP si las concentraciones de AFP estaban elevadas (prueba positiva) y baja si la prueba era normal (prueba negativa).

Tabla III

CONCENTRACIONES DE ALFAFETOPROTEÍNA SÉRICA EN EMBARAZADAS CON Y SIN FACTORES DE RIESGO PARA PARTO PRETÉRMINO

\begin{tabular}{ccccc}
\hline Análisis & $\begin{array}{c}\text { Grupo A } \\
\text { Media } \pm \text { DE }\end{array}$ & $\begin{array}{c}\text { Grupo B } \\
\text { Media } \pm \text { DE }\end{array}$ & t $^{*}$ & Valor $\mathrm{p}$ \\
\hline AFP sérica $(230 \mathrm{ng} / \mathrm{ml})$ & $341,35 \pm 183,51$ & $238,63 \pm 110,46$ & 2,337 & 0,026
\end{tabular}

Grupo A: con factores de riesgo. Grupo B: $\sin$ factores de riesgo. $n=30$ para cada grupo.

*Comparación de medias por T de Student.

Tabla IV

\section{ESTIMACIÓN DE RIESGO DE PARTO PRETÉRMINO EN EMBARAZADAS CON Y SIN FACTORES DE RIESGO CON CONCENTRACIONES ELEVADAS DE ALFAFETOPROTEÍNA SÉRICA}

\begin{tabular}{lcccc}
\hline Análisis/Alternativas & \multicolumn{2}{c}{ Parto pretérmino } & $\begin{array}{c}\text { Odds Ratio* } \\
\text { (IC95\%) }\end{array}$ & $\begin{array}{c}\text { Valor } \\
\mathrm{p}^{* *}\end{array}$ \\
\hline $\begin{array}{l}\text { Con factores de riesgo: } \\
\text { AFP elevada }\end{array}$ & 10 & 04 & 10,833 & 0,008 \\
AFP normal & 3 & 13 & $(1,961-59,835)$ & 1 \\
Sin factores de riesgo: & & & & 1,105 \\
AFP elevada & 3 & 19 & $(0,097-12,473)$ & \\
AFP normal & 1 & 7 & &
\end{tabular}

$\mathrm{n}=30$ para cada grupo. * Análisis de Regresión logística . ** Test de Fischer. 


\section{Tabla V}

\section{CAPACIDAD DIAGNÓSTICA DE LA ALFAFETOPROTEÍNA SÉRICA PARA LA PREDICCIÓN DEL PARTO PRETÉRMINO}

\begin{tabular}{lcc}
\hline Análisis/Alternativas & $\begin{array}{c}\text { Grupo A } \\
\%\end{array}$ & $\begin{array}{c}\text { Grupo B } \\
\%\end{array}$ \\
\hline Sensibilidad (\%) & 76,92 & 75,00 \\
Especificidad (\%) & 76,47 & 26,92 \\
Valor predictivo positivo (\%) & 71,42 & 13,63 \\
Valor predictivo negativo (\%) & 81,25 & 87,50 \\
Predicción total (\%) & 76,66 & 33,33 \\
Prevalencia (\%) & 43,33 & 13,33 \\
Odds pre-test & 0,76 & 0,15 \\
Razón de probabilidad positiva & 3,27 & 1,03 \\
Razón de probabilidad negativa & 0,30 & 0,93 \\
Odds post-test positivo & 2,50 & 0,16 \\
Probabilidad post-test & 71,00 & 14,00 \\
positiva (\%) & & \\
Odds post-test negativo & 0,23 & 0,14 \\
Probabilidad post-test & 19,00 & 12,00 \\
negativa (\%) & & \\
\hline
\end{tabular}

Grupo A: con factores de riesgo. Grupo B: sin factores de riesgo. n: 30 para cada grupo.

Por su parte en el grupo $B$, a pesar de presentar una alta sensibilidad y VPN, que permitiría predecir la ausencia de esta complicación en las embarazadas con resultados normales, la AFP no demostró tener precisión diagnóstica para predecir el PP y al tener razones de verosimilitud cercanas a 1, la probabilidad de presentar o no un PP, no variaría según el resultado de esta prueba (Tabla V y Figura 2 ).

\section{DISCUSIÓN}

Valores elevados de AFP no sólo han mostrado utilidad en el diagnóstico prenatal de defectos del tubo neural y alteraciones cromosómicas (19), sino también como predictor de resultados perinatales adversos, tales como preeclampsia, restricción de crecimiento fetal intrauterino, muerte fetal intrauterina o PP (19-26). En esta serie, las gestantes con FR presentaron significativamente mayores concentraciones de AFP $(p<0,05)$ y prevalecieron las infecciones genitourinarias como principales FR, coincidiendo con lo publicado en otros trabajos, donde se ha señalado que estas infecciones incrementan dos veces el riesgo de presentar PP (27-30). Asimismo, la prevalencia de PP tanto en la totalidad de la muestra analizada como en el grupo

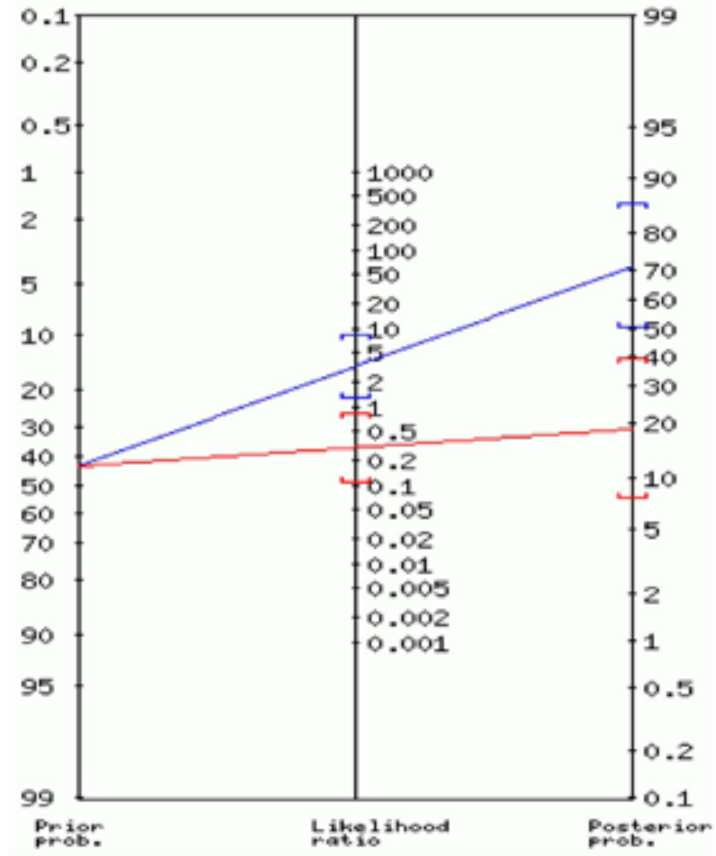

AZUL: TEST POSITIVO ROJO: TEST NEGATIVO 1

Figura 1. Capacidad diagnóstica de la alfafetoproteína sérica para la predicción de parto pretérmino en gestantes con factores de riesgo.

de gestantes con FR resultó mayor que la reportada por otros investigadores (25), quienes determinaron una tasa del $23,4 \%$.

La detección de AFP sérica resultó ser útil para predecir el PP, puesto que demostró tener alta sensibilidad, especificidad, precisión total, post-test positiva, VPP y VPN, resultados que coinciden con los presentados por Salazar y cols (1), quienes encontraron una asociación significativa entre niveles elevados de AFP sérica y PP, con alta sensibilidad, especificidad, exactitud y VPN para su predicción. Cabe acotar que generalmente estos valores son expresados como múltiplos del valor medio (MoM), tomándose como punto de corte 2,5 MoM (21), pero aunque la prueba ha mostrado ser altamente específica, su sensibilidad es baja (31).

Asimismo, se estableció un riesgo significativo de PP, diez veces mayor, en gestantes con FR y niveles elevados de este único marcador, a diferencia de los hallazgos publicados en otras investigaciones (31-33) donde su elevación aislada ha mostrado un riesgo menor y poder predictivo débil para PP. Aunque las embarazadas sin FR y niveles elevados de AFP no presentaron riesgo de PP, ni mostró capacidad predictiva esta prueba, se pudo determinar un alto VPN, lo cual permitiría excluir el 


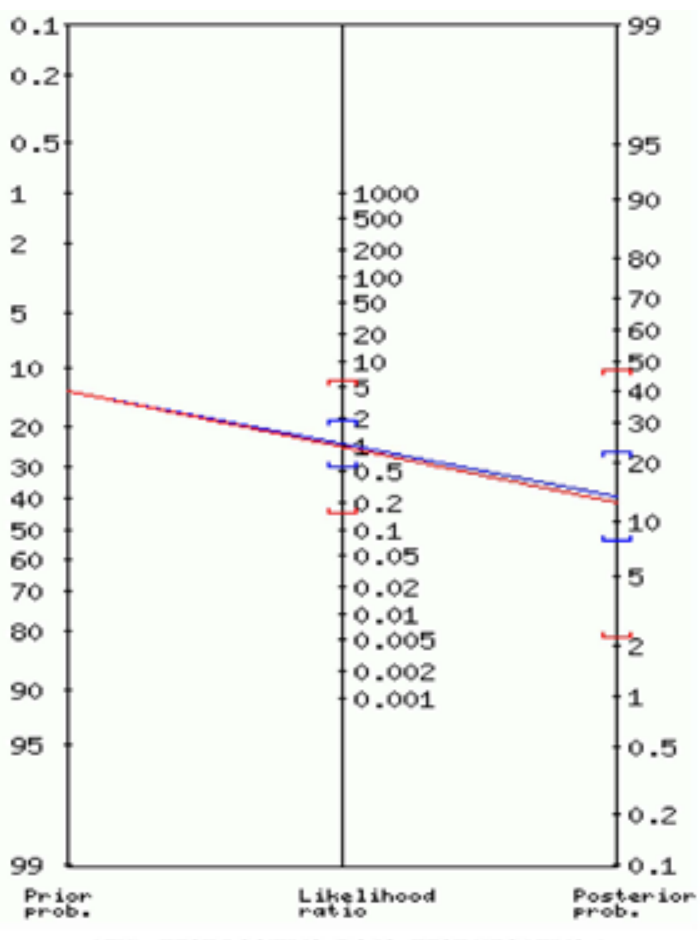

AZUL: TEST POSITIVO ROJO: TEST NEGATIVO 1

Figura 2. Capacidad diagnóstica de la alfafetoproteína sérica para la predicción de parto pretérmino en gestantes $\sin$ factores de riesgo.

riesgo de PP en las pacientes con niveles normales del marcador.

La combinación de AFP con otros predictores como estriol salival (31), laß-hcg $(27,31)$ o la PAPPA (32) ha permitido establecer un mayor riesgo de $\mathrm{PP}$, llegando a casi 10 veces en mujeres con alteración tanto de la AFP como de la PAPP-A (OR: 9,9; IC95\% 4,4-22,0; $p=0,04)$. Al compararse mujeres con AFP elevada (> 2,0 MoM) con niveles elevados de $\beta$-hcg la prevalencia ha sido mayor en gestantes con niveles elevados inexplicados de AFP $(26,5 \%$ vs. 7,3\%), mostrando una elevada razón de verosimilitud para PP al asociarse con una evaluación ecográfica de la función placentaria alterada (34).

Otros autores (33) han demostrado que si asociaban tres marcadores (B-hcg, AFP, e Inhibina A) el riesgo se elevaba a 5,51 (IC95\% 2,23-13,62) mayor probabilidad de presentar PP y el aumento de dos de ellos presentó una alta especificidad y VPN para PP, pero tanto baja sensibilidad como VPP. En otro estudio (27), se determinó que el cuádruple marcador (AFP, B-hcg, estriol no conjugado e inhibina A) tenía valor en la predicción del riesgo de presentar un pronóstico adverso en gestantes de alto riesgo, siendo significativo tanto la alteración de un solo predictor
(OR: 2,8; $p<0,0001)$ como la presencia de dos o más marcadores anormales (OR: 3,2; $p<0,0001$ ).

En este estudio, la AFP demostró mejor capacidad diagnóstica y poder predictivo para el PP entre las gestantes con FR, caracterizado por altas tasas de sensibilidad, especificidad, VPP, VPN y predicción total, asimismo, la prueba mostró mayor $\mathrm{LR}+\mathrm{y}$ menor LR-, lo cual es indicativo de mejor capacidad para predecir la presencia de enfermedad, mostrando esta prueba en las gestantes con FR una alta probabilidad de PP si las concentraciones de AFP estaban elevadas (prueba positiva) y baja si la prueba era normal (prueba negativa). Sin embargo, a pesar de presentar entre las gestantes sin FR una alta sensibilidad y valor VPN, que permitiría predecir la ausencia de esta complicación en las embarazadas con resultados normales, no demostró tener precisión diagnóstica para predecir el PP y al tener razones de verosimilitud cercanas a 1 , la probabilidad de presentarlo, no variaría según el resultado de esta prueba.

Si bien existen limitaciones que imposibilitan generalizar estos resultados al resto de la población, tales como el pequeño tamaño de la muestra y el proceso aplicado para su selección, es aconsejable la evaluación rutinaria de este marcador durante el segundo trimestre, de modo que no sólo permita la detección de malformaciones congénitas, sino también predecir el riesgo de resultados adversos en el embarazo y tomar medidas preventivas oportunas.

\section{CONCLUSIÓN}

Estos resultados demuestran que existe una relación entre la elevación de las concentraciones de AFP y la presencia de PP, mostrando esta prueba tener una alta capacidad diagnóstica y poder predictivo para la ocurrencia de esta patología en embarazadas con factores de riesgo. Por tanto, ante estas evidencias, en concentraciones anormales de este marcador en gestantes con fetos sin defectos congénitos, debe orientar al clínico a la prevención y detección oportuna tanto de PP como de otras complicaciones e integrarlos a la asesoría de riesgo, para evitar las secuelas de las mismas.

\section{REFERENCIAS}

1. Salazar G, Faneite P, Pineda F. Capacidad de la alfafetoproteína en suero materno como marcador predictivo de parto pretérmino. Rev Obstet Ginecol Venez 2009;69 (4):219-25.

2. Burwick RM, Lee GT, Benedict JL, Ross MG, Kjos SL. Blinded comparison of cervical portion length measurements by digital examination vs Cervilenz. Am J Obstet Gynecol 2009;200:e37- e39.

3. Ting HS, Chin PS, Yeo GSH, Kwek K. Comparison of bedside test kits for prediction of preterm delivery: Phosphorylated Insulin-like Growth Factor Binding Protein-1 (pIGFBP-1) test and fetal fibronectin test. Ann Acad Med Singaporei2007;36:399-402. 
4. Villanueva LA, Contreras AK, Pichardo M, Rosales J. Perfil epidemiológico del parto prematuro. Ginecol Obstet Méx 2008;76:542-8.

5. Manrique R, Rivero A, Ortunio M, Rivas M, Cardozo R, Guevara H. Parto pretérmino en adolescentes. Rev Obstet Ginecol Venez 2008;68:144-9.

6. Faneite P, Rodríguez F, Rivera C, Faneite J, Duque J. Estado neonatal en prematurez. 2005-2007. Rev Obstet Ginecol Venez 2008;68:222-7.

7. Faneite $P$, Rivera $C$, González M, Linares $M$, Gómez $R$, Álvarez $L$, et al. Recién nacido de bajo peso. Evaluación. Rev Obstet Ginecol Venez 2002;62:5-10.

8. Cuartas AM. Predicción de trabajo de parto pretérmino espontáneo. Rev Col Obstet Ginecol 2002;53:341-54.

9. Loureiro T, Cunha M, Montenegro N. Avaliação ecográfica do colo uterino e rastreio de parto pré-termo. Método útil ou ainda controverso? Acta Med Port 2006;19:395-404.

10. Torres P, Carrillo J, Rojas J, Astudillo P, Delgado I, Pastén $\mathrm{J}$, et al. Longitud del canal cervical uterino como factor de riesgo de parto prematuro en pacientes sintomáticas. Rev Chil Obstet Ginecol 2008;73:330-6.

11. Iglesias M, Samoroo J, Casanova E, Uzcátegui O. Parto pretérmino: valor predictivo del estriol salival, vaginosis bacteriana y longitud cervical. Rev Obstet Ginecol Venez 2007;67:143-51.

12. Rodríguez N. Fibronectina fetal como predictor del parto pretérmino. Tesis de especialidad. Universidad Centroccidental "Lisandro Alvarado". Facultad de Ciencias de la Salud. Especialidad en Ginecología y Obstetricia. 2009.

13. Gómez E, Castillo C, Villegas Á, Briones JC. Valor predictivo de la fibronectina fetal en amenaza de parto pretérmino. Cir Cir 2004;72:491-4.

14. Mizejewski GJ. Levels of alpha-fetoprotein during pregnancy and early infancy in normal and disease states. Obstet Gynecol Surv 2003;58:804-26.

15. Gagnon A, Wilson RD, Audibert F, Allen VM, Blight C, Brock JA, et al. Obstetrical complications associated with abnormal maternal serum markers analytes. $J$ Obstet Gynaecol Can 2008;30:918-49

16. Mizejewski GJ. Physiology of alpha-fetoprotein as a biomarker for perinatal distress: relevance to adverse pregnancy outcome. Exp Biol Med (Maywood) 2007;232:993-1004

17. Dyce E, Pedreira I, Recio E. Valor de la cuantificación de la alfafetoproteína sérica en embarazos gemelares. Acta Médica de Camagüey 2008;12(1) [Periódico en línea]. Disponible en: <http://scielo.sld.cu/scielo. php?script=sci_arttext\&pid=S1025025520080001000 10\&lng=es\&nrm=iso>. Acceso: 15 de enero de 2012.

18. Salazar G, Faneite P, Pineda F. Valores normales de las alfa-fetoproteína en embarazadas a las 24 y 28 semanas de gestación. Gac Méd Caracas 2007;115:213-8.

19. Salas P, Rodríguez S, Cunningham L, Castro I. Utilidad de la alfa-fetoproteína en el diagnóstico prenatal de defectos del tubo neural y anomalías cromosómicas. Rev Biomed 2003;14:5-10.
20. Yeast JD, Lu G. Biochemical markers for the prediction of preterm delivery. Clin Perinatol 2007;34:573-86.

21. Krause TG, Christens P, Wohlfahrt J, Lei U, Westergaard T, Nørgaard B, et al. Second-trimester maternal serum alpha- fetoprotein and risk of adverse pregnancy outcome. Obstet Gynecol 2001;97:277-82.

22. Costa FDS, Rocha RS, Cunha SPD, Reis FCD, Berezowski AT, Antunes J. Doppler and maternal serum screening in the prediction of pregnancy complications. Radiol Bras 2008;41:7-12.

23. García R, Colín A, Espino S. Alfa fetoproteína como predictor temprano de desenlace perinatal adverso. Ginecol Obstet Mex 2010;78:268-74.

24. Androutsopoulos G, Gkogkos P, Papadopoulos V, Adonakis G, Tsapanos V, Vassilakos P, et al. Mid-trimester maternal serum markers in predicting adverse pregnancy outcome. Clin Ex Obst Gyn 2009;36:23740.

25. Lao MR, Calhoun BC, Bracero LA, Wang Y, Seybold DJ, Broce $M$, et al. The ability of the quadruple test to predict adverse perinatal outcomes in a high-risk obstetric population. J Med Screen 2009;16:55-9.

26. Moína MJ, Cándenas M, Agramunt G, Venta R, Álvarez FV. Utilidad de la alfafetoproteína sérica materna como parámetro de riesgo del embarazo. Prog Obstet Ginecol 2001;44:252-60.

27. Ugalde D, Hernández MG, Ruiz MA, Villarreal E. Infecciones del tracto genital y urinario como factores de riesgo para parto pretérmino en adolescentes. Rev Chil Obstet Ginecol 2012;77:338-41.

28. Jaramillo JJ, López IR, Arango F. Factores asociados con parto pretérmino en el tercer nivel de atención en salud en Manizales. Julio 2004 - Junio 2005. Rev Col Obstet Ginecol 2006;57:74-81.

29. Misra DP, Trabert B. Vaginal douching and risk of preterm birth among african-american women. Am J Obstet Gynecol 2007;196:140.e1-140.e8.

30. Romero C, Chávez A. La actividad sexual como factor de riesgo en la amenaza de parto pretérmino. Rev Fac Med UNAM 2001;44:198-200.

31. Yuan W, Chen L, López A. Is elevated maternal serum alpha-fetoprotein in the second trimester of pregnancy associated with increased preterm birth risk? A systematic review and meta-analysis. Eur J Obstet Gynecol Reprod Biol 2009;145:57-64.

32. Smith GC, Shah I, Crossley JA, Aitken DA, Pell JP, Nelson SM, et al. Pregnancy-associated plasma protein $a$ and alpha-fetoprotein and prediction of adverse perinatal outcome. Obstet Gynecol 2006;107:161-6.

33. Dugoff L, Hobbins JC, Malone FD, Vidaver J, Sullivan L, Canick JA, et al. Quad screen as a predictor of adverse pregnancy outcome. Obstet Gynecol 2005;106:260-7.

34. Toal M, Chaddha V, Windrim R, Kingdom J. Ultrasound detection of placental insufficiency in women with elevated second trimester serum alpha-fetoprotein or human chorionic gonadotropin. J Obstet Gynaecol Can 2008;30:198-206. 